\title{
Study on the Stability and Reliability of the Whole Life Cycle of Tunnel Structure
}

\author{
Youjiang Yang \\ CCCC Second Highway Consultants Co.,Ltd. Wuhang 430056,China
}

Keywords: The entire life cycle; Tunnel structure; Reliability

\begin{abstract}
The tunnel structure life-cycle includes construction period, service period and aging period. Something dangerous maybe happened in the event of stress balance weight distribution of tunnel structure changed and the continuous development of shotcrete and bolt strength during construction. During service period, tunnel structure is more stable but with the influence of earthquake or something like that, the structure risk is back to a relatively high level. After into the aging period, the long-term effects of natural environment and the using environment corrosion lead the structural resistance continued to decline. Due to the environment has rheological effects and the lining was also influenced continuously, which leading to the drop of tunnel structure reliability during this period. Based on the study of the stability of tunnel structure reliability, study on the stability of tunnel structure with full life cycle so as to guide and make sure the safety, applicability, durability and economy of tunnel structural.
\end{abstract}

\section{Introduction}

The structural stability of tunnel has lots of uncertainties on the reliability research in its whole life cycle such as the load, the material parameters, geometric dimensions, the initial conditions and boundary conditions and calculation model. Whether the tunnel structure is safe or not will not only affect the construction progress, quality and cost, but also even related to the personal safety. Therefore, ensuring all the function structure is able to withstand the design within the prescribed time limit and meet the design requirements, which not need too much maintenance ability but can maintain its own performance is critical, that is to ensure the structural safety, applicability and durability, these three aspects constitute the basic content of engineering structure reliability. This paper research and conclude the structural reliability theory and application research at home and abroad, from the tunnel structure reliability theories and methods, the reliability of structural system, structural construction stage and aging stage reliability three aspects, in order to provide guidance to the study the whole life of tunnel structure reliability.

\section{Research on the Theory of Structural Reliability of Tunnel}

It is agreed that the structural reliability is unified, complete the probability structure of power within the prescribed period of time and under specified conditions. Research on reliability theory of tunnel structure, due to the uncertainty of the existing structure design, construction and use of the process, as well as the need to calculate the failure probability of structure in the structural design risk decision theory.

The method of reliability calculation is usually only considered the random variable average value and standard deviation of the two order moment model, reliability by reliability index said. Two order moment model for calculating the structural reliability requires that the expression of the function of the structure is explicit, but usually the structure and function of practical engineering is only a function of a computational process, rather than an explicit expression, then the first order partial derivative of the function can be solved by the finite difference. When the function is implicit, and other reliability solution methods, such as Monte Carlo method and its improved method, stochastic finite element method, response surface method and improved genetic algorithm.

According to the failure conditions of tunnel structure system can be divided into parallel system and series structural systems. The parallel structure system considers that only when every 
component in the structure is failure, the structure of the series is considered that the failure of each component will lead to failure of the whole structure. In engineering practice, the calculation of the tunnel structure system reliability is very complicated, and most are combined in series system and the existence. In order to facilitate analysis, a series parallel structure system is simplified as a series system composed of a plurality of failure modes in practice, so the problem of system reliability engineering in it to the majority of reliability of series system.

Analysis of structural system reliability, we must first find a variety of possible failure modes of structural failure, but even a simple structural model is also very much, all large engineering quantity calculation. In fact, the failure mode, failure probability value was only part of a larger contribution to the failure probability of a structural system, known as the main failure mode, the other can be ignored, so the analysis only considered the main failure modes. After determining the structure of the main failure mode, and then apply multiple failure modes of the reliability calculation method for analyzing structural system reliability is a common way of thinking.

\section{Study on the Stability and Reliability of Tunnel Structure during Construction}

According to the stability theory of motion, the problem of whether or not an object is stable. The failure of excavation face is essentially the strength failure, but when the stress reaches the strength limit of the material, the excavation face in the state of limit equilibrium, the excavation face is prone to failure of instability, the instability criterion:

(1) The convergence criterion. Strength failure belongs to the extreme point instability, its stress-strain curve has the extreme points, the iterative procedure in the calculation of the non convergence. So in the elasto-plastic analysis process, after exclusion of other causes, it is because of the too much development of plastic zone caused by iterative calculation non convergence, can be used as a criterion of instability of the system.

(2) The mutagencity criterion. The system is in the limit equilibrium state that it is changed from one equilibrium state to another, that is to say, the state of the system has changed. The mutation criterion that can reflect the state of the system (such as mutation imagination displacement, yield region Unicom) can be used as a criterion of instability.

For the tunnel excavation face of "steady state", there is no universally accepted, single definition. The stability of the excavation face is evaluated by the limit displacement of excavation face, but in the process of tunnel construction, deformation of excavation surface is not easy to measure. But in the process of construction, the soil pressure on the excavation face can be easily monitored. Therefore, when the chamber pressure is greater than the limit earth pressure on the excavation, excavation face is in a stable state; otherwise, it is in an unstable state; when the chamber pressure is equal to the limit earth pressure, it is in the limit state.

$$
G=g(X)=\sigma_{H}^{R}-\sigma_{H}^{L}=\left\{\begin{array}{l}
>0 \\
=0 \\
<0
\end{array}\right.
$$

The surrounding rock and initial support structure are considered as linear elastic body, and the release rate coefficient by surrounding rock displacement of surrounding rock in the construction process, to simulate the spatial effect of initial support by plane model, in order to improve the efficiency of solving initial support force. Aiming at the initial support structure of the tunnel, the geometrical optimization model of the reliability of the model is a large and feasible region, and the feasible region is small and the constraints are the characteristics of the implicit form, puts forward the calculation method of reliability based on improved genetic algorithm. The accuracy and efficiency of the proposed method is of high reliability, and the method of calculating the other elastic structure is also applicable.

Between tunnel surrounding rock and initial support structure interaction is very complicated, even for deterministic analysis, some reasonable assumptions are usually required to obtain a simplified mechanical analysis model and the mechanical analysis is carried out by using the finite 
element method, which is widely used in engineering practice. The calculation of reliability can be given the explicit limit state equation of bearing capacity of expression in this case will now use the elimination method of non independent variables encountered difficulties. Aiming at this problem, there is usually a penalty function is used to punish the infeasible solutions, in order to get the calculation method of reliability based on improved genetic algorithm.

\section{Research on Reliability of Tunnel Structure System Using}

According to the buried depth of tunnel can be divided into deep buried tunnel and shallow tunnel, the self stability of surrounding rock in deep buried tunnel is relatively good, the safety and reliability than the shallow well, therefore, it mainly introduces the determination method of surrounding rock pressure of shallow buried tunnel. According to the limit equilibrium principle for tunnel surrounding rock of vertical pressure:

$$
\begin{aligned}
& q=h_{1}\left(1-\frac{h_{1} \lambda \tan \theta}{B}\right) \\
& \lambda=\frac{\tan \beta-\tan \varphi}{\tan \beta[1+\tan \beta(\tan \varphi-\tan \theta)+\tan \varphi \tan \theta]} \\
& \tan \beta=\tan \varphi+\sqrt{\frac{\tan \varphi\left(\tan ^{2} \varphi+1\right)}{\tan \varphi-\tan \theta}}
\end{aligned}
$$

There is a certain relationship between the effect of cross section of tunnel lining structure in the lining load $\mathrm{P}$ and the clearance displacement $\mathrm{u}$, load $\mathrm{P}$ in bending cracking, a yielding of the steel bar, and even to reach the ultimate compressive strain of concrete, the structure will remain stable state. Although until the maximum load, displacement and bearing capacity is still some space ,but the tunnel lining structure control of any cross section will fail, may soon lead to the overall structure of the instability. According to the series system to deal with failure section of tunnel lining structure system reliability even conservative, but still feasible. In addition, from operational management department of view, we will not agree to appear as more than a damaged section of the failure mode from the operation and management of department.To sum up, the control section of the cross section of tunnel lining structure of series system, the failure probability of the system is the failure probability of cross section of tunnel lining structure.

For tunnel structural system reliability is calculated in series system reliability. There are several methods of calculation of the series system reliability, numerical integral method, Hohenbichier method, conditions of reliability index method, the equivalent plane method. The numerical integration method is of high accuracy, but it is necessary to carry on the high dimensional integral, and the dimension of the integral is the same as that of the random variable. It is very difficult to compute directly, when the number of random variables is large. Hohenbichier method is an approximate calculation method for structural system in series with a second-order moment method for dimension reduction linearization function, often appear Iterative convergence phenomenon. The accuracy of the reliability index of the system was compared with the method of equivalent plane method and the reliability index of the series system was calculated. The results of the equivalent plane method are most close to the results of the equivalent plane method when the correlation coefficient $\mathrm{P}>0.6$. Therefore, the method of equivalent plane method can be used to solve the reliability of tunnel structure system.

\section{The study on reliability of aging tunnel}

Tunnel structure in the aging stage means that the mechanical properties decrease over time, it has close relationship with the durability of the structure. It is always difficult to make the boundaries between the period of use and age time, there is no fixed structure to terminate the service standard, so you can' t give the beginning and end of the aging time accurately, the research on the reliability of tunnel structure in the aging stage can be used during the aging period and combined in the research. In the general structure reliability theory, the structural reliability is 
defined as the probability of the intended function of the structure, completed within the stipulated time and under specified conditions, it did not consider the attenuation structure resistance over time. However, in the natural environment, the use of the environment and internal factors, along with the secondary lining reinforced concrete come to the aging period, the performance degradation leads to resistance is declining; at the same time, the rheological effect of surrounding rock of tunnel structure dominate well; so reduce the ability of tunnel structure to complete reservation functions in time, the reliability of tunnel structure is reducing. Study the reliability of tunnel structure in the aging period, the durability design and assessment of the tunnel structure, two lining repair and reinforcement, and to determine the service life of the tunnel structure has important significance.

The study on degradation and failure characteristics of tunnel structure performance, deduce the calculating formula of the tunnel structure resistance and ultimate bearing capacity of the corresponding state equation. In view of the existing tunnel in a lot of uncertainty, produce the viscous deformation of surrounding rock as initial elastic deformation, based on elastic finite element theory, the stability of surrounding rock is the evaluation index, the calculation of tunnel lining structure internal force and its standard two different time difference. As to the secondary lining vaults, discuss the concrete strength reduction factor influence on the reliability index, and the reliability of the two lining structure of different section in different time can be obtained to assess, extend the tunnel structure reliability index with time and was almost linear attenuation.

\section{Conclusion}

In the whole life cycle of the tunnel, two factors, structural resistance and load effect, which influence the safety of tunnel structure is changing, and it is affected by the uncertainty and the uncertainty factors, which cause the complexity of tunnel structure reliability change. In the tunnel structure life cycle, consider the tunnel excavation face stability and reliability of initial support, the use of bearing capacity reliability of tunnel structure system reliability, reliability of tunnel structure in the aging were studied. The following conclusions:

(1) Study on the limit support pressure at excavation face of the tunnel; make the different conditions the formation parameters and limit support pressure as a sample, predict a large number of reservoir parameters prediction under the conditions of excavation face limit support pressure ratio, to determine the principle of earth pressure of shield tunneling, the limit state equation is established for the tunnel excavation face stability, create a reliable the method of calculating the series system. In addition to the scientific and reasonable evaluation for the degree of stability of excavation, tunnel construction process to determine reasonable support pressure also has certain reference function to the excavation face.

(2)Starting from the mechanical behavior of tunnel construction, proposes the release coefficient through the displacement of surrounding rock, based on space simulation effect near the excavation surface tunnel using two-dimensional linear elastic model, established the tunnel initial support at different times during the construction period of the reliability index of the feasibility of the model geometry optimization scheme. In the construction process of tunnel excavation under the condition of different rates at different times of the initial support of the reliability index verification according to the proposed method respectively.

(3) For tunnel structural system reliability, it can carry out the following work. First of all, in the geological statistics under the guidance of the theory, the physical parameters of surrounding rock of equivalent deviation increasing function, will simplify the space surrounding rock the rock within the length of the lining of the plane problem. Secondly, the use of statistical theory of brittle rupture, obtain two equivalent lining spatial structure resistance, thus the problem of space structure of the surrounding rock of tunnel length range and reliable for plane problems. Finally, to explore the main failure model of tunnel structure and the reliability calculation method.

(4) According to the existing theory, analysing the degradation and failure characteristics of the tunnel structure performance, deduce the tunnel structure bearing capacity limit state equation is derived. In view of the existing tunnel in much of the uncertainty surrounding rock deformation, 
take the viscosity as initial elastic deformation, elastic finite element analysis based on the theory of surrounding rock, deduced the viscoelastic stochastic finite element incremental formulations, and the preparation of the viscoelastic stochastic finite element incremental program, two time force and calculation of tunnel lining structure standard different time difference. The two lining vault, for an example, to discuss the concrete strength reduction coefficient to bite on the reliability index, and the reliability of the two lining structure of three control section at different time were found to extend the evaluation index of tunnel structure reliability with time and almost linearly. Finally, strengthening the field of tunnel structure aging of observation and experiment, establish the structural resistance is more in line with the tunnel environment attenuation model, the tunnel structure aging reliability assessment more in line with the actual situation, so as to guide the repair and maintenance of the tunnel structure.

\section{References}

[1] Zhihua Li. The whole life process reliability research day of tunnel structure[D], Da lian: Dalian University of Technology, 2007

[2] Yonghua Jiang, Desong Jiang, Minghua Zhao. Loose surrounding rock stability of tunnel structure reliability estimates[J]. Journal of Human University, 2006, 33(2):10-13

[3] Yonghua Su, Xiang Li. Rockbolt and stability of the lining of the tunnel structure reliability calculation[J]. Journal of Civil Engineering, 2011, 44(3):113-118

[4] Nikolaos A. Panayiotou, Sotiris P.Gayialis, I.P. Ilias P. Tatsiopoulos. An e-Procurement System for governmental Purchasing[J]. International Jounal of Production Economies, 2014, 90(1):79-102

[5] Lin Li, Ken Geiser. Environmentally responsible Public procurement(ERPP) and its implications for integrated Product Policy(IPP)[J]. Journal of Cleaner Production, 2015, 13(7):705-715

[6] Shuren Feng, Dingxiang Feng, Xiurui Ge. 3D limit equilibrium method for slop stability and its application[J]. Chinese Journal of Geotechnical Engineering, 2015, 21(6):657-661. 